\title{
Rückblick auf das Jahr 2010: Die FMH war am Ball
}

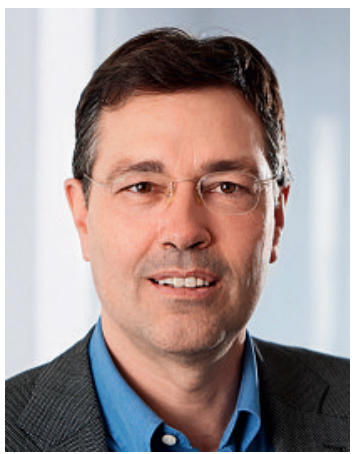

Geht es Ihnen gleich? Haben Sie auch den Eindruck, dass 2010 noch schneller vorbeiging als die Jahre zuvor? Vielleicht liegt es auch daran, dass die FMH in den vergangenen 12 Monaten besonders aktiv gewesen ist.

Sie erinnern sich sicher noch: 2009 haben uns die zahlreichen dringlichen Massnahmen zur Kosteneindämmung im Gesundheitswesen auf Trab gehalten. Doch von den anfänglich 40 Sparmassnahmen sind vor Jahresfrist nur noch wenige übriggeblieben - und einige davon haben im Verlauf des Jahres 2010 sogar noch den Dringlichkeitsstatus verloren. Dass sich diese zum Teil unseligen Massnahmen verhindern liessen, ist auch ein Verdienst der FMH, die viel Aufklärungs-, Lobby- und Interventionsarbeit geleistet hat.

Bundesrat Didier Burkhalter hat andere Prioritäten gesetzt als sein Vorgänger. Statt auf Konfrontation und Einzelmassnahmen zu setzen, versteht er das Gesundheitswesen als Gesamtsystem und zielt auf Kooperationslösungen. Wir hoffen, dass sich diese ganzheitliche Betrachtungsweise durchsetzen wird. Von der Hektik und den zahlreichen Einzelmassnahmen war 2010 schon mal weniger zu spüren.

Praktisch das ganze Jahr hindurch beschäftigte sich die Politik - und damit natürlich auch die Gremien der FMH mit der KVG-Revision, schwergewichtig mit den Themen Managed Care, Wahlmöglichkeiten in der ambulanten Versorgung durch die Patienten, verbesserter Risikoausgleich usw.

Angesichts der stark unterschiedlichen Interessenlage erstaunt es nicht, dass die Revisionsvorlage auch in der Wintersession dieses Jahres wohl noch nicht fertig beraten sein wird - zu wichtig sind diese Grundsatz- und Einzelfragen, nicht nur für die Ärzteschaft, sondern auch für die anderen Stakeholder im Gesundheitswesen. Denn die Auswirkungen der einzelnen Lösungen werden einen beträchtlichen Einfluss auf das Gesamtsystem haben.
Doch 2010 stand für die FMH nicht nur die Politik im Zentrum. Der Berufsverband hat auch eine ganze Reihe guter und für die Ärzteschaft nutzbringender Projekte vorangebracht - hier eine kleine Auswahl:

- Health Professional Card: Bis Ende Jahr hat über ein Drittel der gesamten Ärzteschaft die HPC bezogen. Nun wird es darum gehen, die Einsatzmöglichkeiten für den neuen Ärzteausweis zu erweitern.

- Qualitätsmonitoring: Über 1500 ambulant tätige Ärzte der Hausarztmedizin, Psychiatrie und Orthopädie haben im Rahmen des Projekts Q-Monitoring ihre Qualitätsaktivitäten deklariert. Ziel der FMH ist es, die in der ambulanten Medizin von Ärzten geleistete Qualitätsarbeit schweizweit zu dokumentieren.

- ReMed: Nach einer erfolgreichen Pilotphase hat die FMH das Unterstützungsnetzwerk auf die gesamte Schweiz ausgeweitet. ReMed berät Ärztinnen und Ärzte in Krisen mit einem speziell auf ihre Bedürfnisse ausgerichteten Angebot.

Natürlich dürfen wir die Aktivitäten im Bereich Tarife nicht vergessen. Denn als Berufsverband hat sich die FMH auch 2010 für faire Tarife eingesetzt - sowohl im ambulanten wie auch im stationären Bereich. Brennpunkte waren hier etwa die Besuchsinkonvenienzpauschale, die direkte Medikamentenabgabe, der Labortarif, die TARMED-Revision oder auch die Antragsrunde und Begleitforschung im Bereich SwissDRG.

Am Schluss des Jahres drängt sich die Frage auf: Haben wir alles richtig gemacht, die richtigen Prioritäten gesetzt? Der Rückblick auf das Jahr 2010 macht deutlich, dass die FMH bei den für die Ärzteschaft vitalen Themen rasch agieren kann, dass sie Hand zu Kompromissen bietet und dass sie ihre Positionen wenn nötig auch gegen den Widerstand anderer Gruppierungen vertreten kann.

In diesem Sinne wünsche ich Ihnen und uns allen eine besinnliche Weihnachtszeit, in der wir nicht nur über die wesentlichen Dinge im Leben sinnieren, sondern auch Kraft und Energie für das nächste, sicher nicht weniger anspruchsvolle Jahr tanken können.

Daniel Herzog, lic. iur., MHA Generalsekretär FMH 